\title{
Civilisations
}

Revue internationale d'anthropologie et de sciences

humaines

$50 \mid 2002$

Itinéraires belges aux Amériques

\section{Qui habite la forêt montagneuse de San Miguel Tzinacapan, Sierra Norte de Puebla, Mexique? Éléments de réflexion sur la nature et les apparitions chez un groupe nahua}

\section{Michel Duquesnoy}

\section{OpenEdition}

Édition électronique

URL : http://journals.openedition.org/civilisations/3392

DOI : 10.4000/civilisations.3392

ISSN : 2032-0442

Éditeur

Institut de sociologie de l'Université Libre de Bruxelles

Édition imprimée

Date de publication : 1 décembre 2002

Pagination : 63-77

ISBN : 2-87263-180-1

ISSN : 0009-8140

Référence électronique

Michel Duquesnoy, "Qui habite la forêt montagneuse de San Miguel Tzinacapan, Sierra Norte de Puebla, Mexique ? Éléments de réflexion sur la nature et les apparitions chez un groupe nahua », Civilisations [En ligne], 50 | 2002, mis en ligne le 01 décembre 2004, consulté le 19 avril 2019. URL : http://journals.openedition.org/civilisations/3392; DOI : 10.4000/civilisations.3392 


\title{
QUI HABITE LA FORET MONTAGNEUSE DE SAN MIGUEL TZINACAPAN, SIERRA NORTE DE PUEBLA, MEXIQUE? ELEMENTS DE REFLEXION SUR LA NATURE ET LES APPARITIONS CHEZ UN GROUPE NAHUA
}

\section{MICHEL DUQUESNOY}

\author{
"Parce que dans les yeux des ethnologues sont gravés \\ et imprimés plein de beaux paysages \\ et que sur leur peau sont imprégnés des parfums de fleurs et de bois humide. \\ Aussi la nuit, on entend les musiques et les rires de leurs rêves. \\ On voyage rien qu'à les écouter, " \\ Marianne Scholinchx
}

A Rocío et à Bernabé

Le chamane C.F.F., âgé d'une cinquantaine d'années, nous mit en garde $\mathrm{du}$ coin de la bouche, après avoir planté ses petits yeux vifs dans les nôtres: « Dis-toi bien ceci: il y a autour de nous des êtres de notre monde, mais aussi des êtres qui ne sont pas de notre monde ". ${ }^{1}$ Plus tard, il prévient: "Certains vont jusqu'à se transformer en animal, ou en personne connue. D'autres modifient le paysage. Ils le font pour t'égarer et te faire du mal. " Un conte intitulé: “El Takauatsal, le Sécheur d'hommes» commence par ces mots: "Cet homme-là, les anciens l'appelaient "le Takauatsal »; il déambulait de nuit et il va t'effrayer là où tu habites, il va aller te crier après. Et si tu te mets à courir après lui, eh bien ! c'est lui qui va te suivre, en hurlant, en hurlant jusqu'à t'épuiser... $»^{2}$ Et notre comadre Tayde se met à craindre la vindicte du maître de la montagne, Kuouajtaxiuan, pour un jeune tatou mort en captivité...

Nous voilà prévenus. Ce n'est donc pas à une promenade idyllique dans la belle forêt verdoyante et humide de la basse montagne que cette contribution engage. Pas plus il ne sera question de répertorier la faune et la flore exceptionnelles de la région ${ }^{3}$, malgré tout l'intérêt de la question. Nous dépasserons, en effet, les limites et les brèches de notre réalité pour tenter de découvrir les méandres d'une appropriation du monde fort différente de la nôtre: il s'agira d'écouter les Nahua de la communauté de San Miguel Tzinacapan évoquer les entités extra-humaines diverses qui peuplent les 
environs immédiats de leur biotope. ${ }^{4}$ Place donc pour un petit voyage au pays des lutins et des monstres ...

\section{SAN MIGUEL DANS LA SIERRA NORTE}

Communauté nahua ethniquement assez homogène ${ }^{5}$, dans laquelle se sont mêlés quelques koyomej (métis) dont personne ne songerait à étouffer l'influence, San Miguel compte une population de quelque 4000 têtes réparties sur un vaste territoire, lui-même étagé sur divers paliers situés entre $500 \mathrm{~m}$ et $1000 \mathrm{~m}$ d'altitude. La région se distingue par son climat tempéré humide, chaud l'été, frais l'hiver et par une Nature ${ }^{6}$ généreuse à souhait, parfois exubérante, à la lisière des pineraies d'altitude et de la végétation franchement luxuriante des basses plaines dévalant vers l'Etat de Veracruz. Cet espace favorable a permis très tôt l'établissement de concentrations humaines dont l'indice présent de population s'avère relativement élevé pour le Mexique (entre 80 et 200 habitants $/ \mathrm{km}_{-}$). Actuellement, la frange nord-est à laquelle se rattache notre village doit sa relative prospérité à la culture du café, dont l'introduction remonte au début de la seconde moitié du XIX ${ }^{e}$ siècle. Toutefois, les entreprises d'exploitation forestière de Puebla ou de Mexico ont fait leur apparition voilà peu. Après avoir déclenché une phase de déforestation systématique des cimes les plus élevées, c'est tout l'équilibre géophysique qui risque d'en ressentir les effets pervers - ainsi les glissements de terrain, en septembre et octobre 1999, qui ravagèrent les ranchos situés à quelques dizaines de minutes de marche par rapport au centre villageois.

D'un point de vue culturel, San Miguel compte une écrasante majorité d'indigènes nahua (sans doute $90 \%$ ) et une minorité métisse dont la relative intégration ne s'accomplit pas toujours sans heurt. Les incidents physiques sont rares, voire inexistants de nos jours. Néanmoins la distanciation culturelle anime les moments forts de la dynamique interculturelle communautaire que viennent renforcer divers sobriquets plus ou moins péjoratifs, traces évidentes des inévitables a priori interethniques. Pour les présenter très brièvement, il nous suffira de dire que les divergences principales portent sur le rapport au temps, à l'argent, à la vie communautaire, aux discours du corps et au monde magique. ${ }^{7}$ Enfin, on rappellera que cette zone de la Sierra de Puebla brasse en son sein une frange non négligeable de la population totonaque. Si les interpénétrations culturelles demeurent évidentes, Tzinacapan ne compte selon nos sources - que quatre femmes totonaques (mariées à un époux nahua). Signalons que les Totonaques se voient affublés d'appellations méprisantes telles "nacos" ou "naquitos". Par contre, les versants opposés de la vallée, que plusieurs rios entrecoupent, abritent plusieurs communautés rattachées à cette ethnie. Quelques heures d'une promenade éreintante à travers les herbes et les rocailles recouvertes de mousses glissantes suffisent pour atteindre les beaux villages d'Ecatlán, flanqué sur sa crête calcaire dominant le canyon, ou de Tuzamápan, un rien plus en retrait. C'est dire l'évidente facilité des contacts, aisance entravé de coupures et de suspicions. Les fêtes patronales très hautes en couleurs dont la contrée garde le secret favorisent une masse d'échanges non négligeables. ${ }^{8}$ 
Malgré les difficultés que soulève le désir légitime de la sauvegarde identitaire auxquelles se confrontent ces communautés rurales, on ne peut que souligner combien les traditions affirment un caractère tenace, derrière lequel se retranche, non sans orgueil, une grande partie de la population. Une acculturation émaillée de multiples nuances se laisse appréhender davantage chez les plus jeunes. Ainsi, à San Miguel, si la quasi-totalité des habitants peut s'enorgueillir d'un bilinguisme particulièrement satisfaisant, $c^{\prime}$ est le náhuat qui nourrit les conversations, les rites, les contes et les croustillantes blagues un rien paillardes. Une perception du monde et un univers singuliers distancient notablement nos villageois de Cuetzalan, le chef-lieu de canton tout proche. L'ascendant des métis y a été beaucoup plus érosif. Les êtres fantastiques de la forêt semblent n'appartenir, pour les Cuetzaltèques, qu'à de sombres réminiscences, susceptibles toutefois de reprendre du sens. Ne serait-ce que lors d'une visite chez un curandero qui pourrait évoquer l'une ou l'autre apparition...

\section{LES NAHUA, LA MONTAGNE ET LA NATURE}

Les Nahua de la Sierra Norte ont élaboré une conception de la Nature fort originale par rapport à notre concept occidental. En gros, Nature et culture ne sont jamais confondues, ni entièrement distinctes. Les Nahua exprimeraient davantage une sorte de différence de degré entre la culture et la Nature. Aussi $l^{\prime}$ impact de la modernisation les oblige à reconfigurer subtilement les rapports entre l'une et l'autre. ${ }^{10}$ Pas plus, selon nous, et dans une même logique de pensée, n'expriment-ils jamais une nette distinction entre le "normal" et le "merveilleux", ou encore entre le "sacré" et le "profane", sans pour autant les confondre. Là où l'ethnologue s'emballe pour l'un ou l'autre événement, récit ou conte "merveilleux", son informateur, quant à lui, n'y voit que du "normal" i.e. du quotidien. ${ }^{11}$ Car, en effet, quoi de plus prévisible, pour celui qui s'éloigne un peu trop des sentiers et des passes en forêt, que de croiser l'un ou l'autre être surprenant par sa petitesse, sa force, sa laideur ou son sens de l'humour déconcertant ? S'il peut, c'est sûr, trouver raison de s'inquiéter, notre infortuné promeneur sait que le surnaturel - plutôt que l'anormal - n'est qu'une dimension aussi évidente, aussi réelle que la contingence et l'immédiateté. Le Nahua s'efforcera de conférer un sens à l'événement prodigieux: l'anormal serait de ne pas pouvoir lui en donner! Dès que l'ethnologue acquiert quelque familiarité avec ses informateurs, ceux-ci lui raconteront avec la plus grande simplicité leurs rencontres occasionnelles avec l'un ou l'autre personnage venu de l'“ailleurs", et même - pourquoi pas? - avec le diable en personne. De plus, quand il s'agit de la maladie, de la mort et de l'infortune, le premier besoin manifesté par la victime et par son entourage est la demande d'une explication. Tout événement doit s'insérer dans un discours cohérent sur le monde. Et tout ce petit manège surnaturel peut s'avérer utile... Empirisme, certes! Sans doute! Mais il s'agit ici de relever une sorte d'empirisme sociologique qui permet de calquer sur l'événement redoutable un discours social qui lui demeure inaliénable. 
C'est que, pour les Nahua, l'univers est tissé d'un continuum extrêmement ténu, extraordinairement complexe, qui rattache ce que nous, Occidentaux et universitaires, distinguons comme catégories naturelles et comme catégories magico-cosmologiques. ${ }^{12}$ Cette singulière alchimie donne du sens au monde, aux humains et aux non-humains, sans jamais délaisser ces éléments aux affres d'un hasard par ailleurs totalement absent chez nos Indiens. Car c'est effectivement pour contrer le chaos qu'engendre le manque - ou la perte - de sens que les entités et les hommes sont amenés à se croiser lors de rencontres hautement dramatiques. Elles contribuent à conférer un ordre intelligible au déséquilibre sans cesse menaçant. Elles permettent de comprendre le monde comme parsemé de lieux de passage et d'échange, un monde dans lequel la communication l'emporte sur le cloisonnement. ${ }^{13}$ Ces apparitions dénotent un langage: elles appartiennent à la syntaxe culturelle des Nahua sanmigueleños.

Si nous devons constater chez les Nahua un ensemble de perceptions singulières de la Nature dont l'homme n'est jamais absent, il n'en demeure pas moins que celle-ci est perçue, décrite et ressentie comme une matrice fourmillant de forces grandioses et contradictoires. L'être humain participe, on l'a dit, de cette source souveraine de puissances avec laquelle il entretient des rapports d'échanges. Paradoxalement peut-être, les modalités de ces accords sont mouvantes: rien ni personne ne peut en garantir la permanence. Pourquoi ? Sans doute parce que cet univers de pouvoirs - que nous appelons "nature" n'est ni seulement une Mère généreuse, ni seulement une entité délicate à préserver. Réceptacle de puissances qui ne se laissent appréhender que dans la complexité, certes! Mais dans lequel l'homme participe en tant qu'acteur, que spectateur et que consommateur. Or, qu'il consomme ou qu'il agisse, $c^{\prime}$ est toujours de l'intérieur, jamais de l'extérieur, car, en somme, l'homme appartient pleinement à ce réseau de pouvoirs: essentiellement, il en partage la texture et les composantes, dont il ne se distingue que par des différences ontologiques.

Il n'empêche ! Le Nahua exprime son rapport à la Nature en opposition entre l'espace domestique ${ }^{14}$ (maison, village) et le mont, la montagne ${ }^{15}$, soit "l'endroit où les arbres poussent". ${ }^{16}$ En outre, les endroits cultivés, faiblement habités, extérieurs au village - les ranchos - relèvent du "mont" (kuouajtaj). ${ }^{17}$ Peut-être parce que tous les champs mènent soit aux hauteurs difficilement accessibles et aux cavernes, soit encore vers les cours d'eau. En outre, le faîte des collines et les cours d'eau apparaissent dans la cosmovision nahua comme autant de lieux dangereux d'où surgissent les entités surnaturelles inquiétantes. Peut-être parce que là n'est pas l'espace vital de l'homme ? Peut-être aussi parce que ces endroits sont des voies d'accès du et vers le Talokan, cette espèce $\mathrm{d}^{\prime}$ inframonde administré par les puissants Talokan Tata et Talokan Nana. ${ }^{18} \mathrm{Et}$, que par là, ils libèrent leurs nombreux serviteurs Talokej ; ou encore que, par ces canaux, ils tolèrent le passage des redoutables sorciers nagual. Bien sûr, en principe, les autorités de ce monde-autre sont plutôt généreuses, favorables à l'homme, dont elles reçoivent offrandes et supplications, selon les demandes spécifiques. Ne pourvoient-elles pas à la réussite des récoltes et au peuplement des animaux des bois ? Néanmoins, cette prodigalité des maîtres du Talokan suppose le respect des normes établies. En outre, diverses forces plutôt néfastes 
peuvent se croiser en ces lieux retirés par rapport à la communauté. Ce qui accroît les risques.

Nous mettons là le doigt sur une facette originale de la perception de la Nature par les Nahua de San Miguel: elle prodigue largement ses biens. Plantes et animaux comestibles foisonnent, à l'état sauvage. Souvent, hommes, femmes et enfants reviennent au foyer avec de nombreux fruits, légumes, herbes médicinales qui n'impliquent qu'un seul effort: celui de la cueillette. Quant aux animaux de la montagne propres à la consommation, que d'aucuns n'hésitent pas à chasser ou à pêcher, leur abondance relative tend à imposer des précautions rigoureuses. Deux raisons majeures les justifient: d'abord parce que certains animaux de la montagne prêtent à certains humains leur indispensable alter ego ${ }^{19}\left(\right.$ tonal $\left.^{20}\right)$; ensuite, parce que ces animaux appartiennent aux maîtres du Talokan et qu'ils les libèrent généreusement, sans aucun excès toutefois, toujours jalousement.

Or, si l'activité de la chasse est loin d'être rare - sans être essentielle ${ }^{21}-$, elle suppose le respect des normes de déférence et de régulation établies par les Nahua: un animal de la montagne vient du Talokan ${ }^{22}$, il est un émissaire de Notre-Père et de Notre-Mère-de-l'alimentation-des-gens ${ }^{23}$. Le chasser en état de faute grave, telle l'infidélité conjugale, ou encore déprécier l'animal et l'abandonner, mort ou blessé dans les fourrés sans l'honorer d'une "sépulture", c'est s'exposer à une répression pouvant entraîner la mort. Le plus souvent toutefois, les maitres du Talokan se contentent de restreindre la reproduction des animaux, ou d'infliger un terrible susto (frayeur) au contrevenant. Ainsi, nous avons vu notre comadre blêmir de terreur lorsque le tatou que lui avait rapporté son mari mourut "de tristesse" après quelques jours! Loin même de l'enterrer avec égard, elle préféra le boucaner afin de le manger car, nous affirma-t-elle, "un animal de la montagne mérite notre respect. Si maintenant qu'il est mort, nous ne le mangeons pas, ses parents, ceux du Talokan (los del Talokan) viendront nous le réclamer et nous tomberons malades ou ton compadre aura un accident. C'est sûr ! ${ }^{24}$

Toutefois, selon le mode caractéristique d'opposition basé sur l'ambivalence et la dualité, si la Nature est source d'abondance - c'est son aspect "généreux" -, elle n'en est pas moins une source constante de dangers. L'éloignement du village, puis des champs ou des plantations de café, entraîne un potentiel néfaste non négligeable. Qui s'enfonce dans les bois, dans les canyons ou sur les cimes, prête le flanc à diverses menaces: celle, bien réelle, des animaux sauvages $d^{\prime}$ abord ; celle des éboulements occasionnels ou encore les dérapages fréquents sur les pierres glissantes. Ils sont l'occasion d'entorses, d'écorchures plus ou moins profondes mais aussi de mort. Toutefois, si une incursion en forêt $n^{\prime}$ a effectivement rien d'une sinécure, ce sont les dangers d'ordre magique que l'on redoute le plus, surtout à partir du crépuscule. Cavernes, gouffres et croisements de chemin, autant d'entrées et de sorties menant au monde souterrain. Par là circulent les "vents mauvais" (aires malos, amo kuali ejekamej), des animaux pernicieux et une série de gnomes (duendes) dont les intentions à l'égard des humains sont loin de toujours être avouables. Il faudra s'attendre également à rencontrer les éclairs et les orages eux-mêmes 
(kioujteyomej), les maîtres de l'eau, fomentateurs des éclairs et des vents (atxiuanimej), le Takauatsal déjà cité, le Vieillard de la Montagne (Kuouajtajueuentsin), ou encore le Maître de la Montagne (Kuouajtaxiuan). La liste ne s'arrête pas là tant ils sont nombreux à habiter ou à hanter l'immense espace "naturel" qui encercle le village. ${ }^{25}$

\section{ASPECTS DE LA DEVOTION POPULAIRE A SAN MIGUEL}

Outre les nombreuses mayordomias et le cycle impressionnant des fêtes qui jalonnent le calendrier, coûteuses de multiples efforts - et sur lesquels nous ne pouvons nous étendre ici -, les Nahua de San Miguel entretiennent avec l'orthodoxie catholique des rapports pour le moins élastiques. On pouvait s'y attendre ! Jusqu'à l'aube du XIX siècle, la région n'a été que peu et mal évangélisée en raison des difficultés géographiques et climatiques que rencontraient les missionnaires dans ce secteur de la Sierra de Puebla. En outre, elle échappa durablement au système de sape qu'amenaient avec elles la colonisation et les haciendas (Arizpe 1989 [1973] ; Lupo 1995). Ces circonstances somme toute favorables permirent aux Indigènes d'entretenir un ensemble indéniablement exceptionnel d'aspects singuliers auquel n'ont pu résister de nombreux chercheurs depuis une trentaine d'années. Affirmer ce caractère d'exception ne suppose nullement que cette région a esquivé toute influence, pas plus qu'elle aurait préservé intacts des pans entiers de la culture préhispanique. Nous estimons périlleux autant que malveillant de considérer la cosmovision contemporaine des indigènes comme expression décadente des antiques spéculations méso-américaines ou comme un pâle ersatz de christianisme. En effet, elle n'apparaît jamais comme une simple survivance tant demeurent forts et actifs les ferments de sa crédibilité et de son dynamisme. La cosmovision actuelle des Nahua repose sur un ensemble nouveau de combinaisons puisées dans les deux systèmes qui lui ont donné corps depuis cinq siècles. On parlera alors d'une continuité. Au reste, cette autonomie relative a facilité l'éclosion d'un christianisme indigène expressif que le culte des saints et les fêtes indiennes viennent alimenter. ${ }^{26}$ Les remodelages de l'imaginaire ont donc affecté la masse indigène non sans en respecter pour autant les canons.

De plus, à San Miguel, où ne réside plus aucun prêtre, l'observateur se laisse rapidement séduire par l'efficacité de la dévotion populaire: elle n'a qu'occasionnellement besoin, pour se manifester, de la présence du curé (les actes liturgiques tels la messe, le baptême et le mariage, requièrent un ecclésiastique ${ }^{27}$. Sans porter préjudice à la sincérité des dévots, il est clair que les relations intenses entretenues avec les saints ne le sont qu'en vertu de raisons allogènes au panthéon catholique. Comme ailleurs, la population indigène - et métisse - $s^{\prime}$ accommode du monothéisme occidental en lui agrégeant un cortège de saints, de vierges et de démons aux fonctions souvent éloignées des prémisses dogmatiques chrétiennes. Par ailleurs, le diable, que l'on se plaît à dépeindre sous divers sobriquets, est toujours important. Espèce de trickster d'un genre particulier, craint autant que recherché, amuseur autant que terrible: il est le fourbe qui illusionne. Celui explique qu'il bénéficie malgré 
tout d'une sorte d'attraction ouvertement suspecte aux yeux de tous: pas de culte certes, mais bien des offrandes dont on parle sous le couvert et dont seuls les autres sont réellement coupables. N'est-il pas le riche pourvoyeur des biens faciles enviables par leur absence, hélas, si évidente ? Il ne convient pas de simplement le considérer comme le terme négatif $\mathrm{d}^{\prime}$ oppositions binaires telles bien $/ \mathrm{mal}$; Dieu/diable; etc. Bref, un côté face dénote un catholicisme sincèrement satisfaisant; un côté pile dévoile aux jointures un ensemble d'éléments hétéroclites d'origine bien disparate. L'ensemble valide la cosmovision contemporaine des Nahua en un tout apparemment bien peu systématique. Autrement dit, nous admettons volontiers qu'il subsiste un substrat non négligeable de cette idolâtrie que Gruzinski (1988:194-195) évoque comme la « dimension secrète qui envahit l'essentiel de l'existence indigène ".

Dans cet ensemble où se côtoient et s'entrechoquent sans vergogne sorciers, elfes, saints, pertes de l'âme, Jésus, chamanes, vents néfastes, offrandes, vierges, etc., la confusion n'est qu'apparente. Elle reflète parfaitement les élaborations subtiles de la praxis populaire. Cela complique l'enquête de l'ethnologue qui voudrait trouver une synthèse cohérente. Mais il sait qu'en ce domaine, tout est provisoire, appelé à un constant remaniement fait de rajouts, d'éliminations et de juxtapositions. Il sait que rien n'est figé, que rien n'est dogmatique, surtout pas les rituels ou les cérémonies domestiques auxquels le curandero l'invite. Il remarque que chaque ingrédient, chaque geste, chaque combinaison répond pourtant à un consensus communautaire qui remonte parfois loin dans le temps. Cet assentiment culturel agit un peu comme un canevas indispensable car il est le fruit d'une mutation lente et inéluctable qui a forcé les spécialistes nahua du monde magique à retravailler des thèmes préhispaniques au gré des apports occidentaux, récents ou non. Un peu comme si la subjectivité des chamanes tendait à tisser ou à recentrer les référents collectifs. En ce sens, nous estimons que le chamanisme contemporain des Nahua résulte d'une affirmation du pouvoir particulier des chamanes. Loin des cultes organisés et hiérarchisés de l'ancienne idéologie préhispanique et plus tard, de l'Eglise catholique, ceuxci auraient été amenés, au temps de la colonisation, à interpréter et à intégrer des matériaux nouveaux - ou jusqu'alors inaccessibles car réservés aux seuls prêtres - pour satisfaire les besoins d'une clientèle toujours plus hétéroclite en prise avec les assauts continus de l'infortune, du malheur, de la maladie et de l'exploitation. On pourrait expliquer par là le passage d'un "chamanisme léger" (Duquesnoy 2000:55), inhérent à la puissante centralisation théocratique des Aztèques, à un "ensemble recyclé" (Paradis 1988:99) beaucoup plus vigoureux et généralisé et dans lequel la sorcellerie n'ira qu'en s'affirmant. En un mot, il n'est pas impossible que les curanderos furent parmi les agents les plus efficaces de l'acculturation. De fait, leurs fonctions les placent à la gestion des divers symboles de puissances qu'ils sont censés manipuler. Par là, ils les réinjectent dans le contexte mouvant des cultures indigènes profondément traumatisées suite à la conquête militaire et à l'instabilité générée par l'apport des éléments exogènes puisés au sein de la nouvelle foi, riche d'éléments magiques multiples. L'alchimie de la ferveur populaire, orchestrée et modelée par les chamanes et par tout autre spécialiste des référents symboliques, y trouva son compte: ses 
malheurs et ses besoins d'exaltation religieuse purent y dénicher un sens, un discours novateur sur le cosmos, la nature, les dieux, le corps, la mort. Il serait commun d'affirmer que jamais, cette piété première n'apparaît inerte. En effet, la pratique et le discours des curanderos a permis la conservation d'une partie difficilement quantifiable de la symbolique précortésienne tout en manipulant et en intégrant les données magiques extra-américaines jusqu'à les modifier intensément et donner naissance à un corpus magique particulier. ${ }^{28} \mathrm{Ni}$ entièrement neuf, ni entièrement ancien... mais n'en constituant pas moins une dynamique novatrice.

Dans le monde routinier des communautés indigènes, le prodige est quotidien. Il est, comme l'exprime Gruzinski déjà cité (1985:39), «spontanément, automatiquement intégré dans une grille culturelle qui n'opère pas une distinction catégorique du naturel et du surnaturel. " Parmi ces prodiges, le fait de rencontrer des entités extra-humaines occupe une place appréciable (il ne s'agit pas de vision en état modifié de conscience). Ce qui importe n'est ni la "réalité" ni la matérialité de la rencontre (d'autant que la rencontre a bien lieu !), mais le sens, la signification entière qu'on pourra lui conférer car ces contacts supposent une communication entre les mondes qui n'est jamais perçue à sens unique. Evoquant les margaï, génies importants pour les Hadjeraï, Pouillon (Izard et Smith 1979:50) notait: «(les Hadjeraii) n'y croient pas: cette existence est simplement un fait d'expérience» (nous soulignons). Cette remarque, d'une égale pertinence dans le cas des Nahua, nous permet d'affirmer que l'extraordinaire au quotidien est rendu possible, voire même tangible en ce qu'il est, non seulement prévisible - donc prédélimité -, mais aussi interprétable. En ce sens, l'épisode de l'apparition suscite une démarche intellectuelle visant à décrypter l'événement, à l'intégrer dans une trame socioculturelle et enfin, à dégager une perception optimale du cosmos, de la Nature et de ses forces, sans négliger une conscience singulière du corps, à la fois individuel et collectif. Les apparitions sont un élément fort de la syntaxe culturelle indigène dont les Nahua se servent pour broder leurs relations au réel et au surréel - c'est à dire au monde, selon leurs critères. Nous pourrions écrire: leurs relations aux humains et aux non-humains. Etrange grammaire qui ne cesse de nous surprendre...

\section{OUVRIR OU CONCLURE?}

On pourra légitimement nous en adresser le reproche, mais nous refusons de conclure en cette matière trop malléable: nous gageons que notre contribution laissera un large espace ouvert sur la liberté symbolique que s'arrogent les Nahua au regard de leur préhension du monde fanstasmatique. Celle-ci trahit une richesse de conservations et de réappropriations permanentes qui tentent de protéger l'ensemble communautaire des vertiges du vide et du chaos. Les critères de la conscience nahua dessinent des contours totalement différents de nos réalisations culturelles. N'ont-ils pas raison de permettre à ces voix qui viennent du fond de la forêt de laisser au monde une part appréciable de sa dimension mythique ? Sans doute les qualifierons-nous 
d'imaginaires car nos points de vue et de perception dépendent de vecteurs culturels et philosophiques foncièrement différents. Pour nos amis, l'humain côtoie l'extra-humain. Au premier d'apprendre à tirer parti de l'apparition et de la rencontre. On rappellera que c'est nous, dans notre altérité, qui tentons de dégager un ordre naturel - ce qui est un concept. Il nous devient dès lors loisible d'admettre un monde surnaturel que les spécialistes analyseront en tant qu'illusion ou en tant que réalité non ordinaire. Soit! Mais il reste que ces abstractions sont les nôtres, et qu'elles n'appartiennent pas nécessairement aux Nahua auxquels ces quelques pages tentent de rendre justice. Car, en vertu de leur cosmovision, les limites entre loi naturelle et modalité surnaturelle ne passent pas forcément par les marges que nous dressons pour baliser nos aventures culturelles. C'est dire combien la magie, la sorcellerie et les apparitions relèvent pour eux d'une appréciation particulière qui justifie une autre perception de l'ordre et du désordre cosmique, sociétal et individuel. Seul un scientifique rompu à la logique occidentale peut affirmer que "l'indigène est donc de toutes parts cerné d'éléments "naturels" qui se révèlent à l'examen rien moins que "naturels" " (Gruzinski 1992:38).

Enfin, nous estimons que la tâche principale de l'ethnologue est de rappeler qu'après tout, chaque culture, chaque groupe, chaque individu possèdent les instants et les acteurs du merveilleux qu'ils se donnent avec l'espoir d'en soutirer, au besoin, les valeurs qui rendront possibles les liens étroits (ou lâches, au contraire) qu'ils entretiennent avec l'univers, la Nature et la société. La pensée cosmologique des Nahua avance que la Nature demeure une force vivante, bénéfique et dangereuse à la fois. Logique complexe qui lui confère sa pertinence autant que sa grandeur. Et cette différence radicale nous apparaît comme le ferment principal de sa créativité et de sa vitalité culturelles. Une disparité qui, reconnaissons-le, a prêté à ce peuple les passerelles nécessaires, assorties des inévitables convergences, entre des pratiques et des expressions qui ne sont ni précortésiennes ni européennes. A terme, ces élaborations apparaissent comme authentiquement siennes...

Remerciements

Tout travail ethnologique suppose des dépenses que les déplacements occasionnent. Notre démarche serait impossible sans l'inlassable soutien de Monsieur Daniel Dubuisson, Directeur de Recherches au CNRS. Merci pour sa patience ainsi que pour les bourses qu'il put nous fournir. Nous remercions également Monsieur Jacques Sys, Directeur de l'Institut d'Histoire des Religions, à Lille $3 \mathrm{CDG}$, qui contribua également à encourager nos déplacements. De même, nous désirons exprimer notre reconnaisance à Jocelyne Tousignant, de Montréal, qui a bien voulu lire, commenter et critiquer la première version de cet essai. Tasoj kamatik, siuapiltsin. 


\section{BIBLIOGRAPHIE}

\section{Sigles}

G.R.A.L.: Groupe de Recherches sur l'Amérique Latine.

T.T.O.S.M.Tz.: Taller de Tradición Oral de San Miguel Tzinacapan, Puebla.

U.N.A.M.: Universidad Nacional Autónoma de México

ARAMONI, MA. E.

1988, Bases cosmológicas del shamanismo entre los Nahuas de la Sierra Norte de Puebla (México). G.R.A.L., 13, Université de Montréal.

1990, Talokan tata, talokan nana: nuestras raices. Hierofanías y testimonios de un mundo indígena. Consejo Nacional para la Cultura y las Artes, Dirección General de Publicaciones, I.N.A.H., México.

ARIZPE, L. 1989 [1973] Parentesco y economía en una sociedad nahua. Nican pehua Zacatipan. I.N.I./ Dirección General de Publicaciones, México.

BEAUCAGE, P. (Editeur), 1991, Voces de Tzinacapan. Dimensiones de la cultura náhuat de la Sierra Norte de Puebla, México. G.R.A.L., 23, Université de Montréal.

CEPEC, 1986-1990 Maseual Sanilmej 1-12. Cuentos Indígenas de la región de San Miguel Tzinacapan, Puebla, T.T.O.S.M.Tz., Cuetzalan, Puebla.

CEPEC ET, P. BEAUCAGE, 1988, Maseaulxiujpajmej. Plantas medicinales indígenas. Cuetzalan, Puebla. D.I.F., Puebla.

CHAMOUX, M. N. 1981, Indiens de la Sierra. La communauté paysanne au Mexique.

L'Harmattan, Paris.

DUQUESNOY, $\mathrm{M}$.

1998, Quelques pas avec le chamane nahua de Tzinacapan. Impressions de la Sierra Norte mexicaine. Vivre VI(4):37-42.

2000, Chamanisme, sorcellerie et monde magique au Mexique. Mélanges de Sciences religieuses 57 (2):47-63.

GRUZINSKI, $S$.

1974 Délires et visions chez les Indiens du Mexique. Mélanges de l'Ecole Française de Rome. Série Moyen Âge - Temps Modernes 86: 445-480.

1985, Les Hommes-dieux du Mexique. Pouvoir indien et société coloniale. XVI'-XVII siècles. Editions des Archives contemporaines, Paris-Montreux.

1988, La colonisation de l'imaginaire. Sociétés indigènes et occidentalisation dans le Mexique espagnol XVI ${ }^{e}$ XVIII ${ }^{e}$ siècle. Gallimard, Paris.

1992, La perméabilité des mondes. Rêves et visions dans le Mexique ancien. In Visions indiennes, visions baroques: les métissages de l'inconscient édité par J.-M. Sallmann, pp. 35-56. P.U.F., Paris.

IZARD, M. ET, SMITH, P.1979, La fonction symbolique. Essais d'anthropo-logie. Gallimard, Paris. 
Qui habite la foret montagneuse de San Miguel Tzinacapan,

Sierra Norte de Puebla, Mexique?

KNAB, T. 1991, Geografía del Inframundo. G.R.A.L., 19, Univ ; de Montréal.

LATOUR, B. 1994, Note sur certains objets chevelus. Pouvoir de sorcier, pouvoir de médecin, Nouvelle Revue d'Ethnopsychiatrie 27:21-36. La Pensée Sauvage, Grenoble.

LOPEZ AUSTIN, A.

1989 (1980) Cuerpo humano e ideología. Las concepciones de los antiguos nahuas. 2 vols. UNAM, México.

1995 [1994] Tamoanchan y Tlalocan. Fondo de Cultura Económica, México.

LUPO, A.

1995, La tierra nos escucha. La cosmología de los Nahuas a través de las súplicas rituales. Dirección General de Publicaciones/ I.N.I., México.

1997, Los cuentos de los abuelos. Un ejemplo de construcción de la memoria entre los nahuas de la Sierra Norte de Puebla, México. Anales de la Fundación Joaquín Costa 15:263-284. Huesca.

NATHAN, T. 1993, ... fier de n'avoir ni pays, ni ami, quelle sottise c'était. Principes d'ethnopsychanalyse. La Pensée Sauvage, Grenoble.

PARADIS, L.-I. 1988,Le chamanisme en Méso-Amérique pré-colombienne. Recherches Amérindiennes au Québec, XVIII (2-3):91-100. Montréal.

POUILON, M. 1979, Remarques sur le verbe "croire". In La fonction symbolique. Essais d'anthropologie, édité par M. Izard et P. Smith, pp.43- 51. Gallimard, Paris.

SALLMANN, J.-M. (éditeur), 1992, Visions indiennes, visions baroques: les métissages de l'inconscient. P.U.F., Paris.

SOUSTElle, J. , 1991 [1967] Les Quatre Soleils, Presses Pocket, 8. PLON, Paris.

TOUSIGNANT, J.

1992a, Chiens accompagnateurs et nains ensorceleurs: visages préhispaniques au coeur de deux récits du Nicaragua actuel. Mémoire présenté pour l'obtention du grade de Maître ès sciences en anthropologie. Département d'anthropologie, Université de Montréal.

1992b, Spiritualité amérindienne: la tradition orale du Nicaragua. Religiologiques 6:111-127. UQAM, Montréal.

1994, Mythologie précolombienne et récits nicaraguayens d'aujourd'hui. G.R.A.L., 29. Université de Montréal, Montréal.

T.T.O.S.M.TZ.; BEAUCAGE, P.

1990, Le bestiaire magique. Caractérisation du monde animal par les Maseuals (Nahuats) de la Sierra Norte de Puebla. Recherches Amérindiennes au Québec 20 $(3-4): 3-18$. 
1997, La bonne montagne et l'eau bienfaisante: Toponymie et pratiques environnementales chez les nahuas de basse montagne (Sierra Norte de Puebla). Manuscrit pour Anthropologie et Sociétés 21. Laval. 22 pages.

s.d., The domestication of innovation: the traditionnal Nahua Coffee-Orchard (Sierra Norte de Puebla). Ms.

\section{NOTES}

1 "Dite una cosa muy segura. Hay unos seres mundanos y otros que no son mundanos. Todos nos estan rodeando igual ». C.F.F., Août 1999.

${ }^{2}$ Cepec, T.T.O.S.M.Tz. (1986-1990:7:7). On remarquera que l'illustrateur nahua de ce conte représente le Takauatsal sous les traits d'un être zoomorphe, mi-diable, mi-oiseau.

${ }^{3}$ Le professeur Beaucage de l'Université de Montréal y a réalisé de savantes recherches dont le mérite est de mettre en exergue le savoir indigène en matière de zoologie et de botanique. Nombreuses études publiées à ce jour. Voir Cepec et Beaucage (1988), T.T.O.S.M.Tz.; Beaucage (1992, s. d.).

${ }^{4}$ Un biotope qu'ils connaissent parfaitement! Une randonnée en compagnie d'un Nahua suffit à le remarquer très vite. "The Nahua have developed a very precise knowledge of the different components of their rugged environment (...) all of which is reflected in a rich topographic terminology. " T.T.O.S.M.Tz. ; Beaucage (s.d.: 7). Voir également T.T.O.S.M.Tz. ; Beaucage (1997). Au sujet d'une randonnée: « Découvrir un trésor dans les chants d'insectes et d'animaux, dans les saveurs exquises des fruits sauvages, dans les palabres, dans le ciel brûlant, dans la pluie diluvienne, dans la gamme émeraude de cette nature excentrique, dans le nuage d'évaporation hésitant à abandonner son mouton végétal, dans le serpent tricolore tueur d'hommes. ", Duquesnoy (1998: 39).

5 «... la población indígena, a pesar de seguir mayoritaria (...) sigue manteniendo [su poder] en cierta medida en las comunidades étnicamente más homogéneas, como Tzinacapan. ", Lupo (1997: 265).

${ }^{6}$ Nous employons Nature avec majuscule pour marquer l'espace non habité par l'homme. Nous écartons toute déification de la Nature par les Nahua.

${ }^{7}$ Nous entendons par là le domaine de ce que les universitaires occidentaux appelleraient le monde invisible.

" Yet, a closer look reveals that ethnic differenciation is one of the basic dimensions of social life in the Sierra ». T.T.O.S.M.Tz. ; Beaucage (s.d.: 8).

${ }^{9}$ En ce qui concerne la région de la Sierra Norte, il semble communément admis que les dialectes náhuat(l) remonteraient aux courants migratoires antérieurs à l'invasion européenne. Parmi ceux-ci, l'influence des Toltèques (Altiplano central) et celle des Olmèques-Xicalanèques de Puebla-Tlaxcala semble certaine. Elle expliquerait les variantes du phonème $/-t(l) /$. Les Nahua, quant à eux, affirment parler le mexicano. Selon Arizpe (1989 [1973]:29-30): "En rigor, fueron dos grupos nahuas pertenecientes a dos tradiciones culturales distintas los que inmigraron en el siglo XV: los hablantes de la lengua náhuatl (...) hasta asentarse en las inmediaciones de Huachinango y Zacatlán (...) y los de lengua náhuat (...) ocupan ahora el sureste del macizo ». Nous soulignons.

${ }^{10}$ Nous confessons notre dette à la réflexion suivante de Bruno Latour (1994: 28): “ En fait, l'ordre social et l'ordre naturel n'ont jamais été dangereusement confondus, mais décalés toujours et toujours reconfigurés. " 
Qui habite la foret montagneuse de San Miguel Tzinacapan,

Sierra Norte de Puebla, Mexique?

${ }^{11}$ Jacques Soustelle (1991 [1967]: 69) a bien raison quand il écrit: “ Chez nos Indiens, on ne trouve pas de solution de continuité, de fossé, entre l'une (la physique) et l'autre (la métaphysique). C'est nous qui établissons cette distinction. Elle existe dans notre esprit ». Nous soulignons.

${ }^{12} \mathrm{Ce}$ fin fil explique, selon nous, que les chamanes nahua prêtent aux rêves un caractère tout aussi réel qu'àla réalité événementielle. Les choses visibles dans la contingence sont revêtues d'un voile que le curandero apprend à soulever pour l'outrepasser, grâce à ses alliés, dans le rêve. Notre informateur principal, don L.F.F. nous affirma: « $\mathrm{Si}$ tu ne peux interpréter tes rêves, tu ne peux pas faire du bon travail. " (juillet 1998).

${ }^{13}$ Ceci s'exprime particulièrement bien dans la conviction qu'ont les Nahua selon laquelle les nuages et les vents naissent dans le sein (inférieur) de la terre (aquatique) et qu'ils empruntent les grottes et les cours d'eau, comme béances qui les conduisent au sommet (supérieur) des montagnes où ils peuvent éclater.

${ }^{14}$ Par ailleurs, espace de la femme.

${ }^{15}$ Par ailleurs, espace de l'homme. La division sexuelle du travail et des rapports à la Nature paraît clairement affirmée dans le monde des Nahua de la Sierra. Elle toucherait, en conséquence, la relation au monde magique. En effet, le kuouajtaj demeure un espace redoutable pour les femmes en raison de leurs fonctions reproductives. $\mathrm{Si}$, quotidiennement, elles participent efficacement au ramassage harassant du bois de chauffe ou aux récoltes, il n'est pas de bon ton qu'elles s'aventurent en forêt. L'homme est-il plus proche de la Nature et des forces diverses qu'elle recèle? A première vue, oui ! En outre, il assure des fonctions beaucoup plus brutales, presque physiques, la plupart du temps dans la Nature: il "déchire" la terre, il abat des arbres, etc. Quant à la femme, son domaine d'activité semble davantage "culturel": elle cuisine, éduque les enfants, etc. Si l'homme élague de la matière brute, la femme accommode, transforme, raffine. Voilà qui mériterait sans aucun doute une attention particulière.

${ }^{16}$ En náhuat, kuoujtaj signifie "mont", littéralement "l'endroit des arbres". Ce mot est généralement associé à tepet, "montagne". Voir la planche n¹ dans T.T.O.S.M.Tz. ; Beaucage (s.d.).

${ }^{17}$ Ce qui confirme la spécialisation des activités masculines au domaine brut, sauvage.

${ }^{18}$ Il est utile de préciser que nous basons nos réflexions sur les dires actuels de la population nahua de San Miguel, et principalement sur ce qu'ont bien voulu nous en rapporter les chamanes avec lesquels nous travaillons. Matière non figée par excellence, chacun perçoit et décrit le Talokan selon ses propres connaissances (directes s'il s'agit de curandero). Toutefois, malgré les inévitables variantes, il s'en dégage une sorte de consensus qui témoigne d'une évolution et d'une distorsion évidentes par rapport à ce que d'autres spécialistes ont pu écrire. Voir notamment Knab (1991), Aramoni (1988 et 1990) et López Austin (1995 [1994]). Selon nos analyses, le Talokan, principe bisexué, est à la fois un monde, parallèle à la surface terrestre (taltikpak), plutôt en-dessous, et une entité vivante. Plus qu'un lieu ou un endroit, le Talokan nous est apparu davantage, à considérer nos notes d'enquête, comme un "ailleurs", et s'il nous était permis, comme un "pas ici". "No es un lugar como aquí, poursuit don L.F.F. El Talokan no es un lugar material. Está afuera de la tierra pero está como mezclado a la tierra. " Le fait qu'il est possible pour certains êtres de passer, par les cavernes ou par les voies d'eau courante, de là à ici - et vice versa - n'en rend pas moins cette dimension mystérieuse, fascinante, dangereuse et totalement impropre au développement de l'homme. (Selon C.F.F., "Là-bas, pas question pour toi de traîner en cours de route. L'air y est irrespirable: c'est du "gaz carbonique". Tes poumons seraient brûlés. Mais pour eux qui habitent là-bas, c'est un endroit magnifique. Bon, si tu aimes ce que tu y vois quand tu vas chercher ce que tu attends, c'est ton problème: mais tu peux y rester prisonnier. Pour toujours ! "). Y résident les entités maîtresses des animaux et de la végétation. Tout porte à croire qu'elles administrent la terre, au nom de Tata et de Nana, en tant que source de vie et de pouvoirs. Or si elles pourvoient à toutes les formes de vie, les hommes savent qu'ils en dépendent fortement car ils sont inclus totalement dars ce cycle vital. "Ils sont notre père et notre mère ", affirment les Nahua. En outre, ces derniers se représentent la terre 
comme un être vivant qui voit et qui entend. Elle génère, via Talokan Tata et Nana, les animaux et les plantes, autant que les bonnes récoltes, la pluie et un bon nombre de maladies. C.F.F. assure: « $\mathrm{Si}$ nous nous comportons mal avec cette mère, alors elle nous corrige. Elle ordonne aux serpents de nous punir. Les serpents sont ses fils, de mauvais fils bien sûr (malos hijos) mais ils sont aussi à son service ". C'est dire le caractère ambigu qu'ils entretiennent avec la terre. Précisons davantage. Selon le même informateur, Dieu (Dios Padre) - i.e. le dieu chrétien - vit au ciel. Jamais il ne visite le Talokan dont il laisse la plus entière surveillance à Tata et à Nana. Dios Padre se contente de parfois leur réclamer quelques comptes. Enfin, notre informateur affirme que ces deux gérants n'appartiennent pas à notre monde ("No son mundanos". Faut-il entendre qu'ils appartiennent seulement au semanauak, le "cercle de terre" ?) alors que Dios Padre fait partie intégrante, quoique surpuissante, de notre monde ("Coma vas a creer que Dios Todopoderoso no pertenece a nuestro mundo ? El también es un mundano ! Pero el Talokan no es mundano.") (Interviews réalisées en Septembre 1999). Le rôle de créateurs imparti à Talokan Tata et à Nana (présentés comme des entités éternelles) a été mis en évidence dans un conte rapporté par E. Zamora Islas "Nuestros creadores de Talokan " (Beaucage 1992:8-14). Nos informateurs leur ont toutefois nié formellement la responsabilité de la création. "Solo Dios es creador !" s'exclame don L. "Son mentirosos los que dicen que Nuestro Padre y Nuestra Madre del Talokan son creadores " s'insurge C. Tous deux insistent sur l'aspect généreux de ces donateurs de vie (sic !). Ces divergences - qui n'étonneront aucun ethnologue - témoignent sans doute des processus d'acculturation mis en branle dans cette zone, peut-être comme conséquence inévitable du militantisme catholique romain des membres d'une organisation caritative dénommée PRADE.

${ }^{19}$ Le destin de la personne est intimement lié à son double. Tous les êtres humains ne possèdent pas nécessairement un double animal dans la montagne. Seuls les individus dotés d'un caractère fort pourraient y prétendre (de la "forte tête" au sorcier). Les autres devraient se contenter d'un chat, d'un poulet, ou d'un porc (animaux domestiqués d'origine européenne). C.F.F. nous assure même que chacun de nous possède... sept doubles et qu'il vaut mieux ne pas être sept fois "porc" car ces animaux, dépourvu de caractère, n'ont qu'une vie bien courte. « Si uno es puerco, de qué le sirve vivir ? Al puerco lo criamos para matarle".

${ }^{20}$ On trouve actuellement à San Miguel une prédilection très marquée pour le terme ekauil "ombre". Tonal nous a semblé accablé d'une désuétude que nous ont suggérée nos informateurs ("Ya no decimos asi" $)$. Il suffira ici de noter que le tonal-li désigne $l^{\prime \prime}$ "entité animique" qui meut chaque individu et dont la dépossession entraîne inévitablement la mort. Sur le concept de tonalli dans le Mexique ancien, voir López Austin 1989 (1980): 223-252.

${ }^{21}$ Moufettes, sarigues, coati, tatous, rongeurs, écureuils, etc. Certains animaux sauvages ne sont pas chassés. Les activités cynégétiques ne s'attardent ni aux serpents, ni aux rapaces. Voir T.T.O.S.M.Tz, ; Beaucage (1990).

${ }^{22}$ Certains Nahua estiment que les animaux de la montagne y "descendent" pour dormir ou encore pour y soigner leurs blessures.

${ }^{23}$ Toteiskaltikatotatsin et Toteiskaltikatonantsin.

${ }^{24}$ L'anecdote nous révéla qu'un animal del monte ne peut être simplement jeté à même le sol. « Les animaux viendraient le manger et c'est grave ! ’... et que par contre " si tu le remets en terre, il retourne auprès de ceux qui lui ont permis de sortir [du Talokan-réserve]. Tu ne le vexes et il n'est pas fâché après toi. Il ne cherchera pas à se venger.» Nous vécûmes cet incident en juillet 1999. On remarquera, en outre, que, parmi les animaux domestiques, la négligence de l'homme n'affecte que le chien, le dindon, l'abeille, tous droits présents sur le continent aux temps précortésiens.

${ }^{25}$ Tousignant a analysé le phénomène des apparitions des duendes et des "chiens accompagnateurs" auprès des Nahua du Nicaragua. Voir 1992a, 1992b, 1994. Elle rappelle avec à propos la nature différente de ces entités par rapport aux êtres humains qu'ils côtoient. Pour notre part, nous 
Qui habite la foret montagneuse de San Miguel Tzinacapan,

Sierra Norte de Puebla, Mexique?

tenterons de publier prochainement une recension des divers êtres fantastiques que nous avons recensés à San Miguel Tzinacapan.

${ }^{26}$ Le culte des saints et les fêtes religieuses, principalement patronales, relèvent d'une lente construction qui trahit un ensemble typique de mentalités et de repères indigènes. Elles donnent, à n'en pas douter, un système cohérent au cadre existentiel et social de la communauté. Elle y trouve un cadre revendicatif précieux. Pour illustrer notre affirmation, il suffira de dire que les San Migueleños estiment, non sans malice, que les fêtes de Cuetzalan sont plus fades que les leurs. Rappelons que, de nos jours, la cabecera est aux mains des Métis et que San Miguel est majoritairement indigène..

${ }^{27}$ Protestants et Témoins de Jéhovah représentent une micro-minorité sans réelle incidence notable. ${ }^{28}$ Sur le plan thérapeutique par exemple, - lequel implique toujours une théorie -, les chamanes, on le sait, témoignent de dispositions d'adaptation étonnantes. Mutatis mutandis, qu'il nous soit permis de leur appliquer ce point important dégagé de la clinique ethnopsychanalytique qu'anime Tobie Nathan (1993: 17): “Accepter de "manier" les "théories" qui organisent la pathologie des patients, implique évidemment une reformulation du statut de la "théorie" en clinique, et en premier lieu de la théorie à laquelle adhère le clinicien ". Les guillemets sont de l'auteur. Selon nous, un des grands traits du génie chamanique méso-américain a été d'adapter et de réadapter sans cesse sur un canevas traditionnel (sens fort) les multiples éléments exogènes que la colonisation mit en branle. Voir notre Duquesnoy (2000). 